\section{Nachruf auf Herrn Dr. med. Rainer Amberg, MA}

Am 26.07.2021 ist Dr. med. Rainer Amberg im Alter von 70 Jahren nach langer schwerer Erkrankung in Freiburg gestorben. Er war ein disziplinierter Mensch, von hohem Arbeitsethos und wissenschaftlicher Neugier. Vor allem auch war er ein sehr gebildeter Mensch. Nach seinem Abitur 1972 hat er zunächst Mittelalterliche Philologie (Mittelalterliche Geschichte/Kunstgeschichte/Latein) studiert und war nach Abschluss seines Studiums für 2 Jahre wissenschaftlicher Mitarbeiter am Institut für Geschichte der Medizin am Universitätsklinikum Freiburg. Erst dann entschloss er sich, Medizin zu studieren. Sein Studium finanzierte er durch Arbeit bei niedergelassenen Radiologen in Freiburg. Damit war die Radiologie ein steter Begleiter seines Medizinstudiums, und es wundert nicht, dass er dann in der Rechtsmedizin einen Schwerpunkt in der forensischen Radiologie gefunden hat. Schnell erkannte er auch den Wert der Endoskopie für die forensische Diagnostik, wobei die Netzhaut sein bevorzugtes Untersuchungsobjekt wurde. Er dürfte wohl zu den ganz wenigen Kollegen oder Kolleginnen in der Rechtsmedizin gehören, die reguläres Mitglied einer Ophthalmologischen Fachgesellschaft wurden. Seine Weiterbildungszeit absolvierte er am Freiburger Institut für Rechtsmedizin. Stets sprach er mit Hochachtung davon. In lockerer Anbindung an das Baseler Institut für Pathologie erwarb er solide histologische Kenntnisse. Nicht alle Stationen seiner beruflichen Laufbahn sollen hier wiedergegeben werden. Er war ein unabhängiger Mensch, ein streitbarer Geist.

Herr Amberg zählte zu den wenigen freiberuflichen Rechtsmedizinern Deutschlands. Eine Reihe rechtsmedizinischer Institute sicherte sich seine Mitarbeit, insbesondere Göttingen (2005-2007), langfristig Bonn, Kiel/Lübeck und Gießen/Marburg. Mit diesen arbeitete er auch wissenschaftlich zusammen.

Sein gründliches rechtsmedizinisches Wissen, seine breiten Methodenkenntnisse und sorgfältigen Analysen machten ihn zu einem sehr gefragten und angesehenen Gutachter. Trotz seiner zügigen Arbeit waren seine Gutachten bis ins letzte Detail ausgearbeitet und vorbildlich visualisiert mit den relevanten klinischen Daten, radiologischen, makroskopischen und histologischen Untersuchungsergebnissen.

Er hatte auch für eine Reihe von Semestern einen Lehrauftrag für Rechtsmedizin an der Juristischen Fakultät der Universität Passau inne, was inm große Freude bereitete.

Geprägt war sein Wesen auch von der Liebe zur Musik, genauer zur Barockmusik, zu Georg Friedrich Händel. Davon war er erfüllt und sprach gern über eine große Nähe zur Händel-Gesellschaft in Halle, die über Jahre gewachsen sei.

Mit Herrn Dr. med. Rainer Amberg hat die Rechtsmedizin einen kritischen Geist verloren, der fehlen wird.

\section{K.-S. Saternus, Göttingen}

Rechtsmedizin $2021 \cdot 31: 474$

https://doi.org/10.1007/s00194-021-00527-z

(c) Springer Medizin Verlag GmbH, ein Teil von Springer Nature 2021

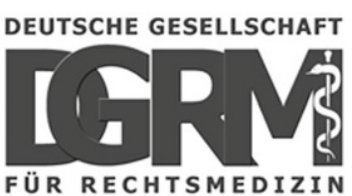

Verantwortlich für den Inhalt der Mitteilungsseiten der DGRM Prof. Dr. Silke Grabherr Institut für Rechtsmedizin Lausanne-Genf (CURML)

Chemin de la Vulliette 4

$\mathrm{CH}$ - 1000 Lausanne 25

silke.grabherr@chuv.ch
Termine rechtsmedizinischer Kongresse entnehmen Sie bitte den folgenden Webseiten oder scannen Sie den QR-Code:

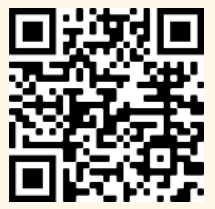

https://www.dgrm.de/tagungen/jahrestagung-dgrm

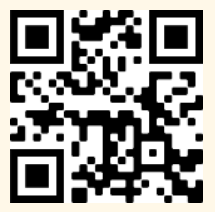

http://www.gmkongresse.de 